\title{
CULTIVO DE MURTILLA (Ugni molinae Turcz.) COMO AL- TERNATIVA DE DIVERSIFICACIÓN PRODUCTIVA PARA LA AGRICULTURA FAMILIAR CAMPESINA DE LA CORDILLERA DE LA COSTA.
}

\author{
Cristian Aguila Chacón ${ }^{1}$, Laura Nahuelhual Muñoz ${ }^{1-2 *}$ \\ ${ }^{1}$ Instituto de Economía Agraria, Universidad Austral de Chile, Casilla \# 567, Valdivia, Chile. \\ ${ }^{2}$ Fundación Centro de los Bosques Nativos FORECOS, Pedro Aguirre Cerda \# 2150, Valdivia, \\ Chile.
}

\begin{abstract}
Murtilla (Ugni molinae Turcz.) husbandry as an alternative for diversification for small farms in the costal range of southern Chile Key words: Murtilla, technical and economic feasibility, subsistence agriculture, Chilean coastal range
\end{abstract}

Murtilla (Ugni molinae Turcz.) is a native shrub endemic in Chile, and the only wild species that has been investigated for productive purposes and domestication. Due to its low soil fertility requirements and its great ability to compete with other species, murtilla could be an interesting option as a fruit crop in southern Chile, particularly in marginal coastal areas commonly dominated by small farmers. The aim of this study was to determine the technical and economic feasibility of cultivating murtilla in the coastal range of the Valdivia Province and compare the resulting financial indicators to those from sheep farming, the main productive activity carried out by small farmers in this area. The feasibility was also investigated under alternative scenarios (self production of murtilla plants, irrigation subsidy, and no exports). The net present value (NPV) in the original scenario and 10 -year time horizon was $\$ 16,329,837 \mathrm{ha}^{-1}$ with an internal rate of return (IRR) of $33.9 \%$. In the alternative scenario, the NPV fell to $\$ 5,076,015 \mathrm{ha}^{-1}$ with an IRR of $25 \%$, indicating the high profitability of murtilla crop in both scenarios compared to sheep farming, which reached a NPV of $\$ 11,222 \mathrm{ha}^{-1}$ and an IRR of $10.8 \%$. The results confirm that growing murtilla

\section{Resumen}

Palabras clave: Murtilla, factibilidad técnica y económica, agricultura familiar campesina, cordillera de la costa

La murtilla (Ugni molinae Turcz.) es una especie arbustiva endémica de Chile y la única investigada con fines productivos y de domesticación. Debido a sus bajos requerimientos de fertilidad de suelo y su gran habilidad para competir con otras especies, la murtilla podría convertirse en una interesante opción frutícola para la zona sur del país, particularmente en zonas marginales costeras que comúnmente corresponden a sectores de agricultura familiar campesina (AFC).

El objetivo del estudio fue determinar la factibilidad técnica y económica del cultivo de murtilla en seis localidades costeras de la Provincia de Valdivia y comparar los indicadores financieros con aquellos de la producción ovina, rubro principal de la AFC en esta zona. Igualmente, se investigó la factibilidad del cultivo en un escenario alternativo (producción propia de plantas, bonificación al riego, venta a mercado doméstico sin exportación). El valor actual de los beneficios netos (VABN) del cultivo bajo el escenario original y un horizonte de tiempo de 10 años, fue $\$ 16.329 .837 \mathrm{ha}^{-1}$ con una tasa interna de retorno (TIR) de 33,9\%. En el escenario alternativo, el VABN disminuyó a $\$ 5.076 .015 \mathrm{ha}^{-1}$ con una TIR de $25 \%$, indicando la alta rentabilidad del cultivo incluso en este escenario, comparado con la producción ovina que alcanzó un VABN de $\$ 11.222$ ha $^{-1}$ y una TIR 
as a fruit crop is a profitable alternative, which can contribute to the productive diversification of small farms in coastal areas of southern Chile. de $10,8 \%$. Los resultados obtenidos confirman que el cultivo de murtilla se presenta como una alternativa rentable, que puede contribuir a la diversificación productiva de la AFC en las zonas costeras del sur de Chile.

\section{INTRODUCCIÓN}

La murtilla (Ugni molinae Turcz.) es una especie arbustiva endémica de Chile perteneciente a la familia de las Mirtáceas. Es una de las frutas silvestres más populares de Chile y la única investigada con fines productivos y de domesticación (Tacón et al., 2006).

Posee una distribución muy amplia encontrándose desde la Región de O'Higgins hasta la Región de Aysén (Tacón et al., 2006), no obstante, la mayor frecuencia de la especie ocurre desde la depresión intermedia ( $\left.72^{\circ} \mathrm{LE}\right)$ hacia la Cordillera de la Costa $\left(74^{\circ} \mathrm{LE}\right)$, entre los $10 \mathrm{y}$ 20 m.s.n.m (Seguel et al., 2000).

La murtilla se adapta bien a suelos de baja fertilidad (Lavín y Vega, 1995; Seguel et al., 2000; Seguel y Torralbo, 2004) presentando además una gran habilidad para competir con otras especies, por lo cual podría adaptarse muy bien a suelos degradados y convertirse en una interesante opción frutícola para la zona sur del país (Torres et al., 1999). Particularmente, debido a sus bajos requerimientos de fertilidad de suelo, la producción de murtilla cultivada se presenta como una alternativa productiva de preferencia para las zonas marginales costeras, que comúnmente corresponden a sectores de agricultura familiar campesina (AFC) (Aguila, 2008).

Las comunidades costeras de la zona sur de Chile en las regiones de La Araucanía (35 -39 L S), Los Ríos (39 - 40 L S) y Los Lagos $\left(40^{\circ}-44^{\circ}\right.$ L S $)$, han recolectado históricamente murtilla silvestre, tanto para la elaboración de alimentos para el autoconsumo, principalmente mermeladas, conservas y licores, como para la generación de ingresos a través de la comercialización local de pequeños volúmenes del fruto y productos artesanales derivados (Aguila, 2008). En los meses de Marzo y Abril algunas familias de estas comunidades costeras, se dedican exclusivamente a la recolección de murtilla. Se estima que el volumen transado a través de las diferentes vías de comercialización, bordea los $800 \mathrm{~kg}$ por recolector en cada temporada, lo que permite generar un ingreso complementario muchas veces relevante. Específicamente, en la costa norte de Valdivia existen alrededor de 30 familias que recolectan un volumen total estimado en 16 toneladas por temporada, el que es comercializado en el mercado local (consumidores finales) y a la pequeña agroindustria (Aguila, 2008).

Aunque la recolección silvestre de esta especie seguirá siendo fundamental en sustentar mercados locales, la consolidación del mercado nacional y sobretodo internacional, requiere cambiar la visión de la murtilla como un producto forestal no maderable (PFNM) a una alternativa de cultivo frutícola. En este contexto, es relevante generar información que apoye la decisión de inversión en el cultivo para distintos estratos de productores, en la medida que existan las condiciones agroecológicas necesarias para el cultivo, como aquellas presentes en la zona costera de la Provincia de Valdivia.

Estudios realizados por el Instituto de Investigaciones Agropecuarias (INIA) por más de 10 años, han permitido proponer de manera preliminar un protocolo técnico para el cultivo de la murtilla en forma comercial ${ }^{1}$. Dicho protocolo contempla todas las etapas de establecimiento y manejo agronómico del cultivo, y ya se ha puesto a prueba con pequeños y medianos productores, principalmente de la región de La Araucanía.

Tomando como base este protocolo de producción, el objetivo de este estudio fue determinar la factibilidad técnica y económica del cultivo de murtilla en la zona costera de la Provincia de Valdivia y comparar los indicadores financieros obtenidos con aquellos generados a partir de la producción ovina, rubro principal de la AFC en esta zona. Igualmente, se estudió 
la factibilidad del cultivo bajo un escenario alternativo donde el agricultor produce sus propias plantas, existe una bonificación al riego y la comercialización es exclusivamente local y regional, no existiendo un mercado exportador.

\section{MATERIAL Y MÉTODO}

\section{Área de estudio y agricultores seleccionados}

La zona de estudio abarcó las localidades rurales de Bonifacio, Las Minas, Curiñanco, El Potrero, Punucapa y Los Pellines (73⒉ - $73^{\circ} 16^{\prime}$ L O y los $39^{\circ} 45^{\prime}-39^{\circ} 42^{\prime}$ L S). El universo de estudio comprendió 63 explotaciones silvoagropecuarias, beneficiarias del Programa de Desarrollo Local (PRODESAL) de la Municipalidad de Valdivia. La forma de muestreo aplicada fue no probabilística o dirigida, por cuanto el interés radicó en seleccionar aquellas explotaciones con mayor potencial y disposición a adoptar el cultivo de murtilla como alternativa de diversificación productiva. De este universo se seleccionaron 15 explotaciones agropecuarias, todas menores a 5 ha de riego básico (H.R.B ${ }^{2}$, gestionadas por pequeños agricultores cuyo ingreso proviene mayoritariamente de la producción silvoagropecuaria y la recolección de PFNM, entre los cuales destaca la murtilla.

El tamaño promedio de las explotaciones es 11,5 ha, superficie cubierta mayoritariamente de bosques secundarios, praderas naturalizadas y matorrales, que mantiene una carga animal promedio de 21,8 cabezas de ovinos y 5,7 cabezas de bovinos. La mayor parte de los agricultores cuenta con título de dominio vigente $(66,7 \%)$ mientras otros $(33,3 \%)$ son herederos (Aguila, 2008). El $93 \%$ de las explotaciones seleccionadas cuenta con una fuente de captación de agua para riego (esteros y vertientes naturales), además de la existencia de una matriz de agua potable rural (Aguila, 2008).

Catorce de los 15 agricultores seleccionados señalaron estar interesados en cultivar murtilla comercialmente en una superficie promedio de 0,36 ha, destinando al establecimiento del cultivo superficies con pradera natural y huertos de baja producción, que en un $50 \%$ de los casos se encuentran cercados perimetralmente (Aguila, 2008).

\section{Recolección de datos primarios y secundarios}

La metodología se centró en la aplicación de una encuesta descriptiva semiestructurada estática a los 15 agricultores seleccionados. La encuesta, aplicada entre Febrero y Marzo de 2007, recabó información respecto a los recursos naturales y productivos existentes en las explotaciones y antecedentes sociodemográficos de los agricultores y sus familias.

Información primaria complementaria se obtuvo a través de entrevistas en profundidad con agricultores de las comunas de Carahue y Puerto Saavedra en la región de La Araucanía, quienes han establecido plantaciones de murtilla y se dedican a la producción de plantas en viveros. $\mathrm{La}$ información recabada en este caso correspondió a datos técnicos y comerciales del cultivo.

La mayor parte de la información secundaria fue proporcionada por el equipo técnico del PRODESAL y la Secretaría de Planificación (SECPLAN) de la Municipalidad de Valdivia. Esta información incluyó la línea base de recursos naturales de los predios y los planes de manejo para optar al Sistema de Incentivos a la Recuperación de Suelos Degradados (SIRSD) de las 15 explotaciones seleccionadas.

Igualmente, se recopiló y analizó información generada por la Asociación Gremial de Productores de Murta (PROMURTA A.G), centrada en aspectos de comercialización y producción de murtilla cultivada ${ }^{3}$.

\section{Análisis y evaluación económica del rubro ovino}

La proyección del rubro ovino para un periodo de 10 años requirió la elaboración de presupuestos técnicos, específicamente, la proyección de la capacidad forrajera de las explotaciones y el desarrollo de la masa ganadera.

Para calcular la cantidad de forraje disponible en las explotaciones analizadas, se utilizó la planilla Excel Balance Forrajero Anual diseñada por INIA-Remehue. Esta planilla es actualmente usada en la elaboración de planes de manejo para las explotaciones agropecuarias de la costa norte de Valdivia. Para esta zona en particular, la capacidad sustentadora de distintos recursos 
Cuadro 1. Capacidad sustentadora de diferentes recursos forrajeros en la costa norte de Valdivia. Table 1. Sustainable capacity for different forage resources on the northern coast of Valdivia

\begin{tabular}{|c|c|c|}
\hline Recurso & $\begin{array}{l}\text { Capacidad talajera } \\
\left(\mathrm{U}^{\mathrm{a}} \mathrm{A}^{\mathrm{a}} \text { ha año }\right. \\
\text {-1) }\end{array}$ & $\begin{array}{l}\text { Rendimiento } \\
\left(\mathrm{kg} \mathrm{MS} \text { ha año }{ }^{-1}\right)\end{array}$ \\
\hline Pradera artificial & 1,3 & 8.000 \\
\hline Pradera mejorada & 1 & 6.000 \\
\hline Pradera natural & 0,4 & 2.500 \\
\hline Nadis & 0,3 & 2.000 \\
\hline Bosques & 0,2 & 880 \\
\hline Matorrales & 0,2 & 880 \\
\hline Frutales & 0,2 & 880 \\
\hline
\end{tabular}

a: Una U.A equivale a un animal bovino de 500 kilos de peso vivo, que consume alrededor de $12 \mathrm{~kg}$ de materia seca (MS) al día (4.400 kg MS año $\left.{ }^{-1}\right)$.

Fuente: INIA, 2007.

forrajeros y su rendimiento se resume en el Cuadro 1.

Para la proyección de la capacidad forrajera se partió de un plan de manejo de las praderas existentes, consistente en mejorar la pradera natural a través de la enmienda calcárea y la fertilización de corrección con fósforo. Posteriormente, la pradera mejorada es fertilizada con una dosis de mantención (60:46:60) siguiendo los planes de manejo realizados por PRODESAL en la temporada 2006 (Aguila, 2008).

Para realizar la proyección de la masa ganadera ovina, el componente bovino de los predios se asumió constante durante los 10 años. Ambos tipos de ganado fueron transformados a unidades animales y en base a su relación porcentual se calculó la disponibilidad de forraje para cada categoría. Las equivalencias en unidades animales (U.A) para distintos tipos de animal fueron aquellas propuestas por Holmes (1980, citado por Olguín, 1997) e INDAP (2007). Una U.A equivale a un animal bovino de 500 kilos de peso vivo, que consume alrededor de $12 \mathrm{~kg}$ de MS al día (4.400 kg MS año ${ }^{-1}$ ).

Se asume que en el año cero se incorporan 45 vientres y 1 macho reproductor, aprovechando al máximo la capacidad talajera existente.
Se utilizaron índices de fertilidad de $120 \%$, reposición de $20 \%$, mortalidad de ovejas y borregas de 5\%, mortalidad de corderos de 12\% (Holmes, 1980 citado por Olguín, 1997) y un porcentaje de desecho de $20 \%$ con una eliminación de 10 ovejas anualmente. La incorporación de borregas provenientes del mismo rebaño tiene como objetivo ajustar la carga animal a la disponibilidad de forraje, aumentando dicha carga desde 10,4 U.A hasta 15,8 U.A en el último año de evaluación.

\section{Análisis y evaluación económica del cultivo comercial de murtilla.}

Para el caso de la murtilla, la evaluación económica se basó en las especificaciones técnicas y de manejo agronómico del cultivo contenidas en el paquete tecnológico para el cultivo de murtilla en forma comercial generado por INIA.

Entre los aspectos más relevantes recomendados por INIA en dicho protocolo están la edad de la planta, la densidad de plantación de estaquillas, las necesidades hídricas del cultivo y la preparación de suelo. Según Torralbo (Com. Pers.), las plantas deben tener entre 1,5 a 2 años para ser llevadas a plantación, tiempo necesario para que desarrollen un sistema radicular eficaz y una vigorosa estructura vegetativa. Las primeras plantaciones comerciales en Chile se encuentran en las comunas de Carahue y Puerto Saavedra en la región de La Araucanía, donde el sistema de plantación se ha realizado en camellones y a una densidad de 3.333 plantas por hectárea usando una cubierta inerte. Según INIA, la distancia de plantación óptima para el cultivo de murtilla es de $3 \mathrm{~m}$ entre hileras y $1 \mathrm{~m}$ sobre la hilera.

El sistema de riego más adecuado es por goteo, y es el que se considera en la presente evaluación. Se asume que los frutos se pueden cosechar a partir del segundo año de cultivo $\left(1,5 \mathrm{t} \mathrm{ha}^{-1}\right)$ y la producción se estabiliza al séptimo año con nueve toneladas por hectárea (Torralbo, Com. Pers.).

La evaluación técnica y económica del cultivo se realizó bajo dos escenarios. El escenario original considera las recomendaciones técnicas de INIA tal como se presentan en el protocolo del cultivo 
y considera tanto la comercialización nacional (mercados locales y agroindustrias) como internacional (exportación vía intermediarios). Bajo este escenario, se asume que un $20 \%$ de la producción se destina al mercado local, un $30 \%$ al mercado nacional (agroindustrias) y un $50 \%$ a exportación. El precio por kilo en el mercado local es de $\$ 525$, equivalente al precio promedio pagado a recolectores en la feria fluvial de Valdivia el año 2006. El precio en el mercado nacional es de $\$ 800 \mathrm{~kg}^{-1}$ correspondiente al precio pagado a recolectores de la costa norte por agroindustria "Productos Mickelsen" en el año 2007. El precio en el caso de exportación es de $\$ 2.193 \mathrm{~kg}^{-1}$, el cual corresponde al precio estimado pagado a productor por PROMURTA A.G., según expectativas de exportación de murtilla para la temporada 2008.

Un segundo escenario (escenario alternativo), que se adapta mayormente a las condiciones existentes en el área de estudio, considera la producción propia de plantas, la existencia de una bonificación al riego y una comercialización restringida al mercado local y regional, destinando un $70 \%$ del volumen producido a agroindustrias y un $30 \%$ al mercado local (Valdivia). En este escenario los precios son $\$ 900 \mathrm{~kg}^{-1}$ y $\$ 525 \mathrm{~kg}^{-1}$, respectivamente. Las plantas son propagadas por los propios agricultores a un costo unitario de \$120 por planta. La bonificación al riego, a través de la ley 18.450 de fomento a la inversión privada en obras de riego y drenaje, subsidia el $75 \%$ de la inversión neta. Cabe destacar que en este caso se asume que el rendimiento disminuye en un $10 \%$ respecto del uso de las variedades de INIA.

Comparación entre ambas actividades productivas

Para poder hacer comparativo el análisis técnico y económico entre el cultivo de murtilla en ambos escenarios y la producción ovina, se tomó como base una hectárea y un periodo de evaluación de 10 años. Para determinar la factibilidad económica de ambas actividades se utilizaron los indicadores valor actual de los beneficios netos (VABN), tasa interna de retorno (TIR), relación beneficio-costo, punto de equilibrio (PE) y periodo de recuperación del capital (PRC).
Se seleccionó una tasa de descuento de $10 \%$ considerando aquella usada en proyectos presentados a INDAP, la cual varía entre $10 \%$ y $12 \%$. Para la pequeña agricultura, rentabilidades de entre $8,5 \%$ y $15 \%$ son bastantes atractivas y reales (Saavedra, 2005). Específicamente, en estudios económicos vinculados a la producción ovina de la región de Los Lagos realizados por diferentes autores, las tasas de descuento empleadas varían entre 9\% y 10\% (Olguín, 1997; Valencia, 1998).

\section{RESULTADOS}

Proyección del rubro ovino y evaluación económica.

La capacidad sustentadora total de los recursos pastoriles existentes en las explotaciones analizadas se calculó en 34,9 U.A año-1 $(153,1$ toneladas de materia seca) siendo las praderas permanentes naturales y mejoradas las que hacen un mayor aporte de forraje soportando 22,3 U.A año ${ }^{-1}$. En la proyección también se consideraron los aportes de las superficies de bosque, frutales y matorrales que son pastoreadas en menor medida por los animales y que en este caso contribuyen con una capacidad talajera de 12,6 U.A año ${ }^{-1}$, la cual se destina en un $29,1 \%$ a los ovinos.

La disponibilidad total de talaje para ovinos aumenta progresivamente debido al mejoramiento de la pradera permanente natural a través de la fertilización, desde una producción de materia seca (MS) de 28,6 $\mathrm{t} \mathrm{ha}^{-1}$ en el año 0 hasta una producción de 53,2 $\mathrm{t} \mathrm{ha}^{-1} \mathrm{MS}$ tha $^{-1}$ en el año

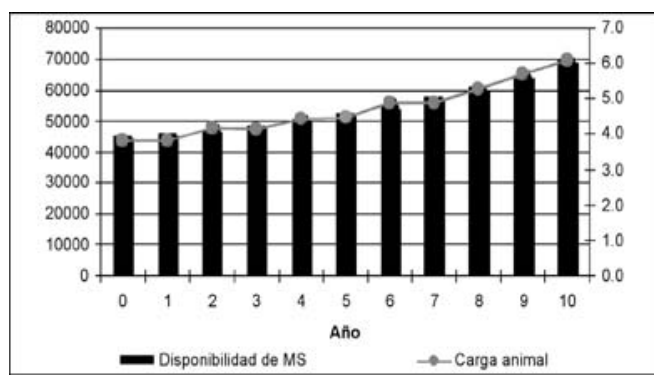

Figura 1. Proyección de la capacidad talajera para el rubro ovino.

Figure 1. Projection of the talajera capacity for sheep production. 
10, considerando una eficiencia de utilización de $75 \%$ (Figura 1). La superficie de pastoreo para ovinos bajo bosque, matorral y frutales, se mantiene constante con una disponibilidad de 16,2 t MS año ${ }^{-1}$ con eficiencia de utilización del $65 \%$.

Tomando como base las proyecciones anteriores, el análisis económico realizado consideró una inversión inicial de \$3.345.000 ha $^{-1}$ que contempla la compra de 45 vientres y un macho reproductor de raza Suffolk, además de la construcción de un galpón de encierro nocturno y de pariciones de $40 \mathrm{~m}^{2}$ con segundo piso para el almacenaje de heno, por un valor de $\$ 1.200 .000$. También se incluye el efecto real que tienen los subsidios en los resultados económicos de las explotaciones analizadas, considerando una bonificación equivalente al $80 \%$ de la inversión neta en infraestructura a través del Programa de Desarrollo de Inversiones (PDI) de INDAP y un subsidio a la fertilización a través del SIRSD equivalente al $80 \%$ del valor neto de la fertilización fosfatada.

Considerando todas las condiciones anteriores, el VABN por hectárea fue de $\$ 11.222$ muy cercano a cero, lo que a su vez indica que la tasa de descuento empleada es muy cercana a la rentabilidad efectiva. La TIR fue de $10,8 \%$ casi equivalente a la tasa de descuento (10\%), situación que se preveía a partir de la magnitud del VABN. Según INDAP (2007), una rentabilidad igual o superior al $8 \%$, determina una escala mínima de operación comercial para el rubro ovino para una explotación campesina ubicada en la región de Los Lagos. Por lo tanto, la rentabilidad de $10,8 \%$ se encuentra dentro de los rangos normales para esta actividad en la zona. La relación beneficio-costo fue de 1,11 indicando que por cada peso invertido y actualizado se recuperan 1,11 pesos generándose una utilidad de 0,11 pesos.

En el primer año del proyecto, los ingresos son muy cercanos a los costos totales y el punto de equilibrio es de 114,7. Esta situación se mejora en el segundo año, donde los costos totales equivalen al 91,4\% de los ingresos brutos. En los años siguientes el punto de equilibrio varía entre 103,9 en el año 3 hasta 88,8 en el año 6 . En el último año (10), se encuentra el punto de equilibrio más bajo del perfil, donde los costos totales equivalen al $72 \%$ de los ingresos brutos generando mayores utilidades, debido en parte a la liquidación de los activos fijos en su valor residual. Lerdón (2001), señala que mientras más bajo sea el punto de equilibrio, el proyecto generará una mayor utilidad, ya que con un menor nivel de actividad cubrirá todos sus costos totales, quedando el remanente como utilidad. El período de recuperación del capital ocurre en el último año del periodo de análisis.

\section{Evaluación económica del cultivo comercial de murtilla bajo el escenario original.}

La inversión necesaria para el cultivo de murtilla considerando estrictamente el protocolo desarrollado por INIA asciende a \$5.404.968 ha $^{-1}$. La mayor proporción de la inversión está representada por la compra de plantas que asciende a $\$ 2.499 .750 \mathrm{ha}^{-1}$ (46\% de la inversión total), asumiendo la compra de plantas de dos años por un valor neto unitario de $\$ 750^{4}$ más un flete de traslado de $\$ 250.000$, desde la comuna de Carahue región de La Araucanía hasta la zona costera en estudio. Estas plantas provienen de variedades silvestres que han sido seleccionados por su calidad de fruta, es decir, que poseen un diámetro ecuatorial por sobre $1 \mathrm{~cm}$, una concentración de azúcares cercano a $16^{\circ}$ Brix y presentan sépalos muy pequeños.

La implementación de un sistema de riego por goteo básico equivale al $21 \%$ de la inversión. El cercado perimetral del huerto equivale a un $13 \%$, mientras que las labores iniciales de plantación corresponden a $17 \%$ incluyendo la preparación de suelo, asesoría técnica, mano de

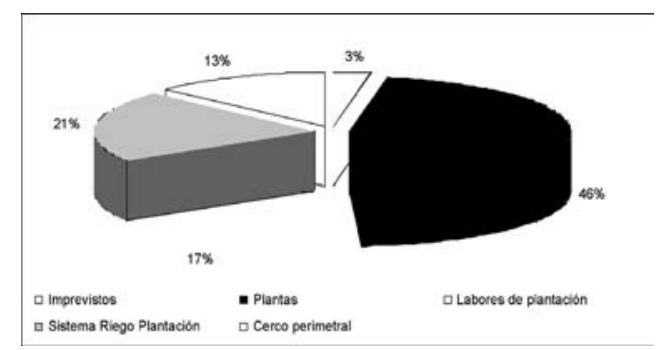

Fuente: Aguila, 2008.

Figura 2. Inversión por hectárea para el cultivo comercial de murtilla.

Figure 2. Inversion per hectare for a commercial murtilla cultivar. 
obra y agroquímicos e insumos aplicados a la plantación (Figura 2).

Los flujos positivos actualizados a una tasa de descuento de $10 \%$ son restados de la inversión, arrojando un VABN de $\$ 16.329 .837 \mathrm{ha}^{-1}$, lo cual indica una alta rentabilidad del cultivo desde esta perspectiva. La TIR correspondiente fue de $33,9 \%$, considerablemente mayor a la rentabilidad general del negocio agrícola en la actualidad, que fluctúa entre 10\% y 15\% (Aguila, 2008). La razón beneficio-costo fue de 2,7 lo cual ratifica la rentabilidad del proyecto.

El punto de equilibrio comienza muy elevado con un $72,3 \%$ de los ingresos brutos cubriendo los costos totales. Sin embargo, va disminuyendo a medida que el proyecto comienza a madurar, bajando a un $11,4 \%$ en el quinto año de cultivo en donde se estabilizan los costos, hasta un 6,6\% en el octavo año cuando la producción del huerto se ha estabilizado. Finalmente, en el último año del proyecto, el punto de equilibrio es de 5,5\% debido a la liquidación de los activos fijos. El periodo de recuperación del capital ocurre en el sexto año de cultivo.

Evaluación económica del cultivo comercial de murtilla bajo el escenario alternativo

En este escenario, donde las plantas para cultivo corresponden a ecotipos locales propagados por los propios agricultores, la inversión inicial disminuye a $\$ 2.105 .707 \mathrm{ha}^{-1}$, un $61 \%$ menos que en el escenario original. Además, en este escenario se descuenta de la inversión inicial el flete de las plantas (\$250.000). La bonificación al riego permite que el costo del sistema de riego disminuya de $\$ 1.137 .835 \mathrm{ha}^{-1}$ en el escenario original a $\$ 284.459 \mathrm{ha}^{-1}$.

En esta situación, el VABN fue de \$5.076.015 ha $^{-1}$, un $69 \%$ menor que en el escenario original. La TIR fue de $25 \%$, mostrando una disminución de 8,9 puntos porcentuales. Sin embargo, la rentabilidad del proyecto continua siendo alta y muy superior a la del rubro ovino. La relación beneficio-costo en este escenario es de 1,5 y el punto de equilibrio al octavo año de evaluación es de 15,4. El capital de inversión se recupera en el séptimo año, un año más tarde que en el escenario original, pero igualmente antes que en el caso de la producción ovina.

\section{DISCUSION}

En el Cuadro 2 se resumen y comparan ambas actividades productivas para los dos escenarios del cultivo de murtilla. La actividad ovina presenta un VABN de \$ $11.222 \mathrm{ha}^{-1}$, muy inferior al del cultivo de murtilla tanto en el

Cuadro 2. Comparación de los indicadores económicos más relevantes en el rubro ovino y el cultivo de murtilla en ambos escenarios.

Table 2. Comparison of the most important economic indicators for sheep production and murtilla cultivation.

\begin{tabular}{|l|c|c|c}
\hline \multicolumn{1}{|c|}{ Indicador económico } & Ovinos & $\begin{array}{c}\text { Murtilla: escenario } \\
\text { original }^{\mathbf{a}}\end{array}$ & $\begin{array}{c}\text { Murtilla: escenario } \\
\text { alternativo }^{\mathbf{b}}\end{array}$ \\
\hline VABN $(10 \%)\left(\$ \mathrm{ha}^{-1}\right)$ & 11.222 & 16.329 .837 & 5.076 .015 \\
\hline TIR $(\%)$ & 10,8 & 33,9 & 25 \\
\hline Relación beneficio-costo & 1,11 & 2,7 & 1,5 \\
\hline PE año $8(\%)$ & 89,7 & 6,6 & 15,4 \\
\hline Inversión inicial $\left(\left(\$\right.\right.$ ha $\left.^{-1}\right)$ & 3.345 .000 & 5.404 .968 & 2.105 .707 \\
\hline PRC (Año) & 10 & 6 & 7 \\
\hline
\end{tabular}

VABN: Valor actual de los beneficios netos; TIR: Tasa interna de retorno; PE: Punto de equilibrio; PRC: Período de recuperación del capital.

a: cultivo siguiendo estrictamente las recomendaciones de INIA y la comercialización incluye el mercado exportador.

b: el agricultor produce sus propias plantas de murtilla, existe una bonificación al riego, y la comercialización 
escenario original $\left(\$ 16.329 .837 \mathrm{ha}^{-1}\right)$ como en el escenario alternativo $\left(\$ 5.076 .015 \mathrm{ha}^{-1}\right)$. Lo anterior se ve reflejado en una TIR de $10,8 \%$ para el rubro ovino, la cual es triplicada por el cultivo de murtilla en el primer escenario y más que duplicada (25\%) en el escenario alternativo. A su vez, los indicadores de beneficio-costo, punto de equilibrio y periodo de recuperación de capital respaldan la factibilidad económica del cultivo de murtilla bajo las condiciones del área de estudio y los supuestos técnicos y comerciales de ambos escenarios.

Los niveles de rentabilidad del cultivo de murtilla en ambos escenarios resisten una disminución considerable de los ingresos, llegando a precios límites (aquellos que hacen el VABN igual a cero) de $\$ 592 \mathrm{~kg}^{-1}$ en el caso de venta a agroindustrias (manteniendo constante el precio de mercado local en $\$ 525 \mathrm{~kg}^{-1}$ ) y de $\$ 400$ $\mathrm{kg}^{-1}$ en el caso del mercado local (con un precio de venta a agroindustrias de $\left.\$ 645 \mathrm{~kg}^{-1}\right)$.

Sin embargo, además de los indicadores de factibilidad económica, se deben tener en cuenta otras variables tanto económicas como sociales y culturales propias del productor que pueden favorecer o limitar la adopción de un nuevo cultivo como la murtilla (Ortiz y Dulcic, 1989).

Los costos de inversión, periodos de recuperación asociados a la inversión en la nueva tecnología, y el acceso a recursos de inversión (créditos) están entre los factores más críticos señalados en la literatura. Particularmente la inversión inicial, si no es subsidiada, puede simplemente hacer inviable el establecimiento del cultivo (Aguila, 2008) a lo cual se suma el hecho de una tardía recuperación del capital invertido. En el presente estudio la inversión que implica el establecimiento del cultivo de murtilla asciende a $\$ 5.404 .968 \mathrm{ha}^{-1}$ y $\$ 2.105 .707$ $\mathrm{ha}^{-1}$ en el escenario original y alternativo, respectivamente, comparado con $\$ 198.750 \mathrm{ha}^{-1}$ en el caso del rubro ovino. Los agricultores que han establecido huertos comerciales en la región de La Araucanía, plantean que la murtilla es una alternativa frutícola viable siempre y cuando existan subsidios estatales, precisamente debido a los altos montos de inversión inicial y el período de recuperación del capital invertido (Aguila, 2008). En este sentido es relevante el acceso de los productores a instrumentos específicos de fomento a la innovación productiva o fuentes financieras expeditas. En el presente estudio se asume por ejemplo la existencia de un subsidio al riego del cultivo y a la asistencia técnica por parte de INDAP a través de PRODESAL.

La disponibilidad de información sobre una nueva tecnología es fundamental en la velocidad de adopción de la misma. En este sentido es relevante el rol de INIA en el desarrollo de la investigación conducente a estrategias mejoradas de manejo del cultivo de murtilla e igualmente el de los asesores técnicos (INDAP y PRODESAL) quienes son los más indicados para transferir y adaptar los protocolos de manejo agronómico del cultivo a las características de una determinada zona y de sus agricultores.

La disponibilidad de mano de obra puede ser también un factor crítico en el éxito del cultivo. En el área de estudio ésta no sería una limitante seria. En promedio cada familia cuenta con 2.7 jornadas hombre por día (160 por temporada) para un requerimiento de 360 jornadas por hectárea y por temporada (Marzo y Abril) en plena producción del huerto a partir del séptimo año. Por otra parte, los meses de cosecha de la murtilla coinciden con meses de bajo requerimiento de mano de obra en las demás actividades prediales.

En huertos comerciales de mayor tamaño, la mano de obra utilizada para la cosecha puede provenir de la utilizada en la cosecha de arándanos, porque ya existe una experiencia y especialización de los temporeros en la recolección de bayas pequeñas y además existe un pequeño desfase entre ambas cosechas (PROMURTA A.G, 2005).

La habilidad operativa de manejo y capacidad administrativa del agricultor son factores que se citan como críticos para la AFC en general. En este sentido, diversos autores señalan que tanto la edad como la escolaridad son factores que influyen en la capacidad de gestión. En el presente estudio, los agricultores tienen en promedio 50 años de edad con niveles de escolaridad que varían entre la enseñanza básica incompleta (27\%) y la enseñanza media completa (33\%). Al respecto Monardes et al. (1993), señalan que una mayor edad y nivel de educación pueden influir positivamente en 
el proceso de adopción de nuevas tecnologías, debido a que se asocian a una mayor experiencia y preparación, que hace a los agricultores más arriesgados y capaces de entender mejor las nuevas técnicas de producción. Por otra parte, el grado de organización de los agricultores facilita el proceso de asociatividad, el cual es fundamental para el desarrollo de la AFC, ya que mejora el acceso a capacidades técnicas y a la innovación (Halabi, 2004).

La disponibilidad efectiva de los insumos necesarios y la existencia de mercado para el producto son factores menos limitantes en el presente caso. La murtilla no requiere de insumos complejos y la mayor parte de ellos está disponible a nivel local. Sin embargo, se debe destacar que si bien los mercados para este fruto han tendido a expandirse, la comercialización, al menos a nivel local, continúa siendo considerablemente informal con precios relativamente fluctuantes.

El mercado de la murtilla está en su etapa de consolidación y se espera que crezca en la medida que el fruto y sus derivados sean conocidos en otros mercados, tanto a nivel nacional como internacional. Un papel importante deben desarrollarlo las empresas que están utilizando el fruto y hojas de murtilla, en materia de publicidad y estrategias de marketing con el propósito de posicionar la murtilla y sus derivados como productos distintivos del sur de Chile (PROMURTA A.G, 2005).

\section{CONCLUSIONES}

Comparado con el rubro ovino, el cultivo comercial de murtilla tiene una inversión inicial alta $\left(\$ 5.404 .968 \mathrm{ha}^{-1}\right)$, muy similar a la de otros berries, aún cuando el agricultor produzca sus propias plantas en cuyo caso la inversión baja en un $61 \%$.

El VABN del cultivo bajo el escenario original y un horizonte de tiempo de 10 años, fue $\$ 16.329 .837 \mathrm{ha}^{-1}$ con una tasa interna de retorno (TIR) de 33,9\%. En el escenario alternativo, el VABN disminuyó a \$5.076.015 ha ${ }^{-1}$ con una TIR de $25 \%$, indicando la alta rentabilidad del cultivo en ambos escenarios en comparación con la producción ovina que alcanzó un VABN de $\$ 11.222 \mathrm{ha}^{-1} \mathrm{y}$ una TIR de $10,8 \%$.
A partir de estos resultados se puede concluir que el cultivo comercial de murtilla se presenta como una alternativa productiva con potencial económico, que puede contribuir al aumento de la diversificación productiva de la AFC de la zona costera del sur de Chile. Sin embargo, es necesario tener en cuenta que la rentabilidad es uno de varios aspectos que determinan la adopción de un nuevo rubro por parte de los agricultores e igualmente deben considerarse las dificultades que representan la alta inversión inicial del cultivo y el período de recuperación del capital.

\section{AGRADECIMIENTOS}

Deseamos agradecer al Sr. Alexis Palma Ingeniero Agrónomo Jefe Técnico del Programa de Desarrollo Local (PRODESAL) de Valdivia por su valioso apoyo en el trabajo en terreno asociado a la presente investigación, y al Sr. Luis Torralbo Ingeniero Agrónomo, Coordinador de la Unidad de Apoyo a Proyectos de Facultad de Ciencias Agrarias de la Universidad Austral de Chile y Gerente del Consorcio Tecnológico Apícola, por sus valiosos comentarios en cuanto a la producción y comercialización del cultivo de murtilla.

También agradecemos la asistencia editorial brindada por la Srta. Brenda Román y la Srta. Alejandra Carmona.

\section{BIBLIOGRAFÍA}

AGUILA, C. 2008. Evaluación económica comparativa del cultivo comercial de murtilla (Ugni molinae Turcz.), respecto del rubro central de la Agricultura Familiar Campesina (AFC) de la costa norte de Valdivia. Estudio de caso. Tesis Lic. Agronomía. Valdivia, Universidad Austral de Chile. 136p.

CHILE, INSTITUTO DE DESARROLLO AGROPECUARIO (INDAP). 2007. Estrategia regional de competitividad por rubro. "Ovinos X región”. Documento de trabajo (On line). <http://indap.iie.cl/fileadmin/template/PROA2008/ planes_estrategias_regionales/ovino_2007/ OVINOSXREGION.doc>. (4 marzo 2008).

HALABI, R. 2004. En la Agricultura Familiar Campesina ¿Por qué es necesaria la asociatividad? 
Nuestra Tierra 230:36.

LAVIN, A., VEGA, A. 1995. Caracterización de frutos de murtilla (Ugni molinae Turcz.) en el área de Cauquenes, Chile. Agricultura Técnica 56:64-67.

LERDON, J. 2001. Formulación y evaluación de proyectos agrícolas y agroindustriales. Apuntes de clases para el curso Formulación y evaluación de proyectos agrícolas. Instituto de Economía Agraria. Universidad Austral de Chile. Valdivia. 146 p.

MONARDES, A.; COX, T., NAREA, D.; LAVAL, E.; REBOLLEDO, C. 1993. Evaluación de adopción de tecnologías. Santiago, Chile. Centro de estudios para América Latina sobre desarrollo rural, pobreza y alimentación, 151p.

OLGUIN, R. 1997. Evaluación técnica económica de un modulo ovino para pequeños productores en la precordillera de la Provincia de Valdivia (Punahue). Tesis Lic. Agronomía. Valdivia, Universidad Austral de Chile. 149 p.

ORTIZ, C.; DULCIC, C. 1989. Innovación tecnológica agropecuaria. Centro Universitario de Desarrollo (CINDA). Proyecto gestión tecnológica. Santiago. Chile. 98 p.

PROMURTA, A.G. 2005. El berry nativo del Sur de Chile. Boletín divulgativo. Proyecto FDI Instituto de Investigaciones Agropecuarias (INIA).
Ministerio de Agricultura. Santiago. Chile.

SAAVEDRA, M. 2005. Evaluación económica de un proyecto de Olivos. Revista Agroeconómico 89:6-9.

SEGUEL, I.; PEÑALOZA, E.; GAETE, N.; MONTENEGRO, A., TORRES, A. 2000. Instituto de Investigaciones Agropecuarias INIA Carillanca. Agro Sur 28 : 32-41.

SEGUEL, I.; TORRALBO, L. 2004. Murtilla: El berry nativo del sur de Chile. Revista Tierra Adentro 57:20-25.

TACÓN, A.; PALMA,; J., FERNANDEZ, V.; ORTEGA, F. 2006. El mercado de los Productos Forestales No Madereros y la conservación de los bosques del Sur de Chile y Argentina. Red de PFNM. Fondo mundial para la naturaleza (WWF). Santiago. Chile. 95p.

TORRES, A.; SEGUEL, I.; CONTRERAS, G.; CASTRO, M. 1999. Caracterización físico química de frutos de murta (murtilla) Ugni molinae Turcz. Agricultura Técnica 59:260-270.

VALENCIA, M. 1998. Evaluación técnica y económica de un módulo de ovinos para pequeños agricultores de la Cordillera de la Costa, décima región. Tesis Lic. Agronomía. Valdivia, Universidad Austral de Chile. 131 p. 